ARTIGO

Recebido em: 06/10/2017

Aceito em: $22 / 05 / 2018$

\title{
Rankings universitários internacionais e o desafio para as universidades brasileiras
}

\author{
University rankings and the challenge for the Brazilian universities
}

\author{
Samile Andréa de Souza VANZ (samilevanz@terra.com.br) * \\ Andres Pandiella DOMINIQUE (andres.pandiella@uc3m.es) ** \\ María Luisa LASCURAIN SÁNCHEZ (mlascura@bib.uc3m.es) *** \\ Elías SANZ CASADO(elias@bib.uc3m.es) ****
}

*Professora da Universidade Federal do Rio Grande do Sul, Departamento de Ciências da Informação, Programa de Pós-graduação em Comunicação e Informação.

** Pesquisador da Universidad Carlos III de Madrid, Laboratorio de Estudios Métricos de Información.

*** Professora da Universidad Carlos III de Madrid, Laboratorio de Estudios Métricos de Información.

**** Professor da Universidad Carlos III de Madrid, Laboratório de Estúdios Métricos de Información.

\section{Resumo}

Os rankings universitários ganharam importância no cenário nacional e internacional e constituem um dos instrumentos de análise do desempenho das universidades. Esta pesquisa analisa o desempenho de 29 universidades brasileiras classificadas nos rankings Leiden, Shanghai, QS e THE. Os resultados obtidos permitem concluir que o desempenho das universidades brasileiras é muito bom, especialmente no que diz respeito ao volume de produção científica, no entanto, o impacto, medido pelas citações recebidas, ainda constitui um grande desafio. 0 volume de publicações nas revistas Nature e Science, assim como a ampliação da colaboração internacional, são estratégias que podem ampliar o impacto e abrir caminho para o alcance de melhores posições nestes rankings. Palavras-chave: Universidades brasileiras. Leiden Ranking. Ranking de Shanghai. Ranking QS. Ranking THE.

\begin{abstract}
The university rankings have gained importance in the international and national scenario and constitute one of the performance analysis mechanisms of the universities. This research analyzes the performance of 29 Brazilian universities rated at the Leiden, Shanghai, QS and THE rankings. The results obtained allowed to conclude that the Brazilian universities performances is very good, especially in terms of the amount of scientific output, however, the impact, measured by the citations, still constitutes a vast challenge. The amount of papers at the journals Nature and Science, as well as the international collaboration, are strategies that can expand the impact and pave the way for reaching better positions at these rankings.
\end{abstract}

Key-words: Brazilian university; Leiden Ranking. Shanghai Ranking; QS Ranking; THE Ranking.

v. 23, n. 53,2018 . p. $39-51$

ISSN 1518-2924 


\section{INTRODUÇÃO}

O setor universitário é um dos principais motores do desenvolvimento científico e econômico de muitos países. No Brasil, grande parte dos cientistas trabalha em universidades públicas e institutos de pesquisa (LETA; GLANZEL; THIJS, 2006; PEDROSA; CHAIMOVICH, 2015), fator que torna fundamental a análise do desempenho dessas instituições. Uma das formas atuais de analisar o desempenho das universidades são os rankings universitários, que constituem importantes ferramentas de avaliação para auxiliar nas políticas de desenvolvimento de C\&T de instituições e países. 0 lançamento do Ranking de Shanghai em 2003 chamou a atenção das universidades, dos governos e da mídia para este tema e representou o início do desenvolvimento dos indicadores para outros rankings universitários internacionais, como o Leiden Ranking, QS University Ranking, e o The Times Higher Education (THE). No Brasil, o jornal Folha de São Paulo apresenta anualmente o Ranking Universitário Folha (RUF). Também em nível nacional, foi lançado em 2014 o Brazilian Research Ranking, que propõe a mensuração das atividades desenvolvidas não só nas universidades, mas também nas instituições de pesquisa brasileiras (VOGEL et al., 2014).

Os rankings universitários internacionais mais importantes, denominados league tables, utilizam para suas avaliações os indicadores básicos de produção científica e reputação, coletados respectivamente através de bancos de dados internacionais de artigos científicos e pesquisas de opinião com acadêmicos e empregadores. Estima-se que os rankings universitários internacionais cobrem uma percentagem entre $1 \%$ e $3 \%$, o que corresponde a cerca de 200 a 500 universidades, dentre um total aproximado de 17.000 universidades do mundo (RAUHVANGERS, 2011; HAZELKORN, 2013).

Os rankings universitários estimularam o debate sobre a qualidade e a performance dos sistemas de educação superior, e geraram um grande impacto na sociedade e na internacionalização das universidades (DE FILIPPO et al., 2012; SANZ CASADO, 2015). 0 rápido crescimento e aceitação dos rankings pela sociedade constitui a prova de sua utilidade, e sua popularidade deve-se em grande parte à simplicidade de seus indicadores (HAZELKORN, 2013).

Apesar das inúmeras possibilidades de uso dos rankings, é importante considerar as críticas recorrentes na literatura, entre elas, a escolha de indicadores baseados fundamentalmente na produção científica, o que coloca todas as outras atividades (como ensino e extensão) em posição de menor importância (ENSERINK, 2007). Outras críticas dizem respeito à capacidade dos indicadores utilizados pelos rankings para medir qualidade; se as diferenças entre os scores obtidos realmente refletem diferenças na qualidade das universidades; e o fato dos mesmos parâmetros serem utilizados para medir atividades de instituições muito diversas (RAUHVANGERS, 2011; HAZELKORN, 2013; SANZ-CASADO et al., 2013; BORNMANN; MOYA-ANEGÓN, 2014). O Manifesto de Leiden corrobora com as críticas e pontua como princípio que "o desempenho deve ser medido de acordo com a missão de investigação da instituição, grupo ou pesquisador" (HICKS et al., 2015, p. 429, tradução nossa). As diferenças de cobertura das bases de dados utilizadas no que diz respeito às áreas do conhecimento (VAN RAAN, 2005) e ao idioma (SAFON, 2013) também são críticas recorrentes na literatura.

Entender as limitações e o que medem os rankings nacionais e internacionais é fundamental para ajustar o desempenho e melhorar o posicionamento das universidades brasileiras (FRANÇA, 2015; SANTOS; NORONHA, 2016). Muitos esforços são feitos para apresentar informação objetiva baseada em números, mas sabe-se que a posição ocupada por cada instituição depende diretamente dos indicadores escolhidos para compor cada um dos rankings. Neste sentido, este artigo objetiva analisar a metodologia dos rankings universitários Leiden, Shanghai, QS e THE e avaliar o desempenho das universidades brasileiras nas edições de 2016. Ao final, são propostas algumas estratégias para enfrentar o desafio dos rankings internacionais.

O Leiden Ranking é publicado anualmente desde 2008 pelo Centre for Science and Technology Studies (CWTS) da Universidade de Leiden, na Holanda. A edição de 2016 
apresenta resultados para 842 instituições de pesquisa baseados em indicadores de produção científica, impacto e colaboração da Web of Science.

O Academic Ranking of World Universities (ARWU), conhecido como Ranking de Shanghai, é produzido pelo Center for World-Class Universities (CWCU), da Shanghai Jiao Tong University. O Ranking avalia mais de 1200 universidades e classifica as 500 com maior desempenho acadêmico e de pesquisa, através de seis indicadores que medem a produção científica; o número de pesquisadores com elevado nível de citações; os antigos alunos ou professores que receberam Prêmio Nobel ou Medalha Fields (seu equivalente em Matemática); e por fim, a publicação nas revistas Nature ou Science. 0 sexto indicador agrega os anteriores e os pondera pelo número de professores da instituição com dedicação em tempo integral (ACADEMIC RANKING OF WORLD UNIVERSITIES, 2016).

O QS University Ranking é produzido pela Quacquarelli Symonds, empresa britânica especializada em educação e estudos no exterior. Após rompimento com o Times Higher Education (THE-QS) em 2009, a empresa passou a produzir seu próprio ranking a partir de 2010. A edição 2016 do Ranking QS avaliou mais de 4 mil universidades e ranqueou as 916 melhores universidades do mundo (QS, 2016).

O Times Higher Education World University Rankings (THE) foi criado em 2004, em cooperação com a empresa britânica Quacquarelli-Symonds (QS), que permaneceu até 2009. A edição 2016-2017 lista 980 universidades, representando 5\% das universidades mundiais, e se autointitula o maior ranking league table, o único a julgar universidades em suas missões fundamentais: ensino, pesquisa, transferência de conhecimento e internacionalização (THE, 2016; SAFON, 2013).

As seções a seguir apresentam a metodologia utilizada pela pesquisa incluindo os aspectos metodológicos mais importantes de cada ranking. Em seguida, os resultados principais são apresentados e discutidos, e a última seção apresenta a conclusão e as estratégias propostas para enfrentar o desafio dos rankings internacionais.

\section{PROCEDIMENTOS METODOLÓGICOS}

Estão presentes neste estudo todas as 29 universidades brasileiras que figuram em ao menos um dos quatro rankings analisados: Leiden, Shanghai, QS e THE. Os dados foram coletados entre maio e novembro de 2016 através da consulta às informações públicas e divulgadas no site dos rankings. Foram buscados resultados para "Brazil" na opção de busca por país nas edições completas de 2016 dos quatro rankings. Os dados do Leiden Ranking foram importados diretamente do site em planilhas Excel, já os dados dos Rankings de Shanghai, QS e THE, disponibilizados em interface web, foram copiados manualmente e organizados em planilha Excel. Posteriormente, os dados foram analisados com o software XLStat e as análises descritivas são apresentadas em gráficos e tabelas.

Tendo em vista que os dados disponibilizados pelos rankings são consolidados, não há problemas aparentes relacionados aos nomes das universidades brasileiras. No entanto, a falta de padronização de nomes institucionais é uma limitação inerente às fontes de dados utilizadas pelos rankings, seja ela WoS ou SCOPUS.

O Leiden Ranking é o único dentre os quatro que divulga seus dados brutos integralmente, ou seja, é possível acompanhar o volume de produção científica, citação e colaboração ao longo da série histórica para todas as universidades. Em contrapartida, Ranking de Shanghai, QS e THE divulgam apenas a pontuação gerada pelos rankings a partir dos dados brutos obtidos pelas universidades. Por este motivo, buscou-se na Web of Science o volume da produção científica das universidades, citações recebidas e artigos publicados nas revistas Nature e Science. A lista dos pesquisadores mais citados foi consultada em novembro de 2016 no Highly Cited Researchers, banco de dados mantido pela Clarivate Analytics. A busca ocorreu pelo nome das universidades brasileiras em estudo.

Dados acerca do número de professores das universidades brasileiras foram coletados em julho de 2016 no Sistema de Informações Georreferenciadas (Geocapes), banco de dados mantido pela Coordenação de Aperfeiçoamento de Pessoal de Nível Superior (http://geocapes.capes.gov.br/geocapes2/).

Os dados de pontuação disponibilizados pelos rankings foram estimados a partir do cruzamento com dados da Web of Science, do Highly Cited Researchers e do Geocapes. Desta forma, universidades listadas pelo Leiden Ranking tiveram seus dados estimados para o 
Ranking de Shanghai, possibilitando uma ideia dos requisitos que precisam ser cumpridos para que estas sejam incorporadas por este ranking.

Algumas especificidades no procedimento para coleta e análise de dados são detalhadas para cada ranking, conforme se descreve a seguir. 0 Leiden Ranking apresenta os dados brutos do volume de artigos e citações. Neste estudo, optou-se pela coleta dos dados inteiros (não fracionada). Para avaliação da produção científica utilizou-se o indicador sizedependent $(\mathrm{P})$, que corresponde ao número bruto de publicações. Já para avaliação do impacto e colaboração, utilizaram-se os indicadores size-independent (PP), que permitem a relativização dos dados em relação volume de publicação da universidade (CENTRE..., 2016).

O Ranking de Shanghai apresenta as pontuações de cada universidade, calculadas a partir dos dados brutos de cada instituição ponderados em relação àquela com maior desempenho em cada indicador, que recebe 100 pontos. São considerados para cálculo os seguintes indicadores e respectivo peso: a) Alumni $10 \%$, representa o número de alunos ganhadores do Prêmio Nobel e Medalhas Fields; b) Award 20\%, corresponde ao número de membros da instituição premiados pelo Prêmio Nobel e Medalha Fields; c) HiCi 20\%, equivale ao número de pesquisadores mais citados em 21 categorias de assuntos do Essential Science Indicators da Clarivate Analytics; d) N\&S 20\%, representa o volume de artigos publicados nas revistas Nature e Science durante os cinco anos anteriores; e) PUB $20 \%$, número total de artigos indexados nas bases Science Citation Index-Expanded e Social Sciences Citation Index no ano anterior. Ao calcular o número total de artigos de uma instituição, um peso especial (peso 2) é atribuído aos artigos indexados pelo Social Sciences Citation Index para compensar a baixa representatividade desta área do conhecimento na base de dados; f) PCP 10\%, indicador calculado com base nas pontuações ponderadas dos cinco indicadores anteriores dividido pelo número equivalente de acadêmicos de tempo integral da instituição (ACADEMIC RANKING OF WORLD UNIVERSITIES, 2016).

O QS University Ranking combina seis indicadores de performance para avaliar pesquisa, ensino, empregabilidade e internacionalização das universidades, e concede $40 \%$ de sua pontuação para a reputação das universidades, medida através de questionários enviados aos acadêmicos pela internet, e 10\% para a reputação perante os empregadores, medido através de questionários enviados a esse grupo. Para a edição 2016 foram entrevistados mais de 70 mil acadêmicos e mais de 35 mil empregadores. 0 indicador qualidade de ensino é mensurado através da proporção de estudantes por professor. 0 indicador de impacto é medido através das citações recebidas no período de cinco anos na base de dados Scopus, dividido pelo número de docentes da universidade.

A base de dados Scopus também é fonte do Ranking THE para mensuração da pesquisa nas universidades, indicador que compõe 30\% do score. Somam-se a ele os indicadores ensino (30\%), citações (30\%), perspectiva internacional $(7,5 \%)$ e transferência de conhecimento da indústria $(2,5 \%)$. 0 Ranking THE também utiliza pesquisa de opinião sobre o prestígio e reputação das instituições de ensino, reunindo mais de 10 mil respostas provenientes de 133 países.

\section{RESULTADOS E DISCUSSÃO}

Um total de 29 universidades brasileiras figuram nos rankings universitários Leiden, ARWU, QS e THE. O Ranking de Shanghai é o mais elitista, por incluir indicadores de prêmios Nobel, Medalha Fields e pesquisadores mais citados, e inclui apenas seis universidades brasileiras, conforme detalhado no quadro a seguir: 
Quadro 1 - Desempenho das universidades brasileiras nos rankings universitários internacionais 2016

\begin{tabular}{|c|c|c|c|c|}
\hline UNIV & $\begin{array}{l}\text { LEIDEN } 2016 \\
\text { (842 univs)* }\end{array}$ & $\begin{array}{l}\text { Shanghai } 2016 \\
\text { (500 univs)* }\end{array}$ & $\begin{array}{l}\text { QS } 2016 \\
\text { (916 univs)* }\end{array}$ & $\begin{array}{l}\text { THE } 2016 \\
\text { (980 univs)* }\end{array}$ \\
\hline Univ de São Paulo (USP) & 11 & $101-150$ & 120 & $251-300$ \\
\hline Univ Est de Campinas (UNICAMP) & 193 & $401-500$ & 191 & $401-500$ \\
\hline Univ Fed de Minas Gerais (UFMG) & 316 & $301-400$ & $551-600$ & $601-800$ \\
\hline Univ Fed Rio de Janeiro (UFRJ) & 213 & $301-400$ & 321 & $601-800$ \\
\hline $\begin{array}{l}\text { Univ Fed Rio Grande do Sul (UFRGS) } \\
\text { Univ Est Paulista Julio de Mesquita }\end{array}$ & 255 & $401-500$ & $461-470$ & $601-800$ \\
\hline Filho (UNESP) & 192 & $301-400$ & $501-550$ & $601-800$ \\
\hline Univ Fed Paraná (UFPR) & 578 & & $701+$ & $601-800$ \\
\hline Univ Fed Santa Catarina (UFSC) & 531 & & $701+$ & $601-800$ \\
\hline Univ Fed São Paulo (UNIFESP) & 368 & & $501-550$ & $601-800$ \\
\hline Univ Fed Ceará (UFC) & 687 & & $701+$ & $801+$ \\
\hline Univ Fed Pernambuco (UFPE) & 598 & & $701+$ & $801+$ \\
\hline Univ Fed Santa Maria (UFSM) & 774 & & $701+$ & $801+$ \\
\hline Univ Fed São Carlos (UFSCAR) & 636 & & $651-700$ & $801+$ \\
\hline Univ Fed Viçosa (UFV) & 764 & & $701+$ & $801+$ \\
\hline Univ Fed Fluminense (UFF) & 678 & & $701+$ & $801+$ \\
\hline Univ de Brasília (UNB) & 656 & & $601-650$ & \\
\hline Univ Est Rio de Janeiro (UERJ) & & & $701+$ & $801+$ \\
\hline Univ Est Londrina (UEL) & & & $701+$ & $801+$ \\
\hline $\begin{array}{l}\text { Pont Univ Católica do Rio de Janeiro } \\
\text { (PUC RJ) }\end{array}$ & & & $501-550$ & $601-800$ \\
\hline $\begin{array}{l}\text { Pont Univ Católica do Rio Grande do } \\
\text { Sul (PUC RS) }\end{array}$ & & & $701+$ & $601-800$ \\
\hline $\begin{array}{l}\text { Univ Fed Bahia (UFBA) } \\
\text { Pont Univ Católica de São Paulo (PUC }\end{array}$ & & & $701+$ & $801+$ \\
\hline SP) & & & $501-550$ & \\
\hline Univ Fed do ABC (UFABC) & & & & $601-800$ \\
\hline Univ Fed Goiás (UFG) & & & & $801+$ \\
\hline Univ Fed Ouro Preto (UFOP) & & & & $801+$ \\
\hline Univ Fed de Lavras (UFLAVRAS) & & & & $801+$ \\
\hline Pont Univ Católica do Paraná (PUCPR) & & & & $801+$ \\
\hline Univ Est Maringá (UEM) & & & & $801+$ \\
\hline Univ Fed Rio Grande do Norte (UFRN) & & & & $801+$ \\
\hline
\end{tabular}

Nota: a posição no Leiden Ranking refere-se ao indicador volume de produção científica (P)

* número total de universidades avaliadas por cada ranking

Fonte: dados da pesquisa

Observa-se no Quadro 1 a proporcionalidade entre o número total de universidades analisadas pelos rankings e o volume de universidades brasileiras. Shanghai, que ranqueia apenas as 500 melhores, reúne o menor número de universidades brasileiras (seis). Logo após encontra-se o Ranking Leiden, que reúne 842 universidades em sua edição 2016 (16 brasileiras); seguido pelo QS, com 916 universidades (22 brasileiras) e pelo THE, o mais abrangente deles, que reúne 980 universidades ( 27 brasileiras).

Algumas das instituições mais antigas do país, como a Universidade Federal do Rio de Janeiro (UFRJ) e a Universidade de São Paulo (SCHWARTZMAN, 2015), figuram entre as mais bem ranqueadas universidades nacionais. 0 Quadro 1 reúne inúmeras universidades paulistas, o que pode ser consequência do investimento de $1 \%$ do produto interno bruto 
(PIB) estadual em pesquisa (FUNDAÇÃo DE AMPARO À PESQUISA DO ESTADO DE SÃO PAULO, 2011) ou uma simples vantagem histórica, já que a pós-graduação brasileira teve início na região Sudeste (CASTRO, 1986). Indubitavelmente as diferenças históricas na criação e desenvolvimento das universidades brasileiras influenciam o desempenho nos rankings universitários internacionais, tendo em vista que a idade das universidades se relaciona ao seu tamanho e este é um aspecto que exerce influencia nos rankings (SAFON, 2013).

Conforme apresentado no Quadro 1, o Leiden Ranking incluiu 16 universidades brasileiras na edição 2016, três a mais que as edições de 2015 e 2014. A Tabela 1 apresentada a seguir está ordenada pelo volume de artigos (segunda coluna) publicados. A quarta coluna apresenta o percentual da produção da universidade que integra o grupo das $10 \%$ publicações mais citadas na WoS (PP Top 10\%). A sexta coluna apresenta o percentual da produção da universidade que integra o grupo das $50 \%$ publicações mais citadas na WoS (PP Top 50\%). Na oitava coluna apresenta-se o percentual de publicações em colaboração institucional (PP Colab), seguido pelo percentual de colaboração internacional (PP Int Colab) na décima coluna. As colunas indicadas pelo cabeçalho Classificação (Clas.) apresentam a classificação ocupada no ranking dentre o total de 842 universidades, em relação ao indicador da coluna esquerda.

Tabela 1 - Universidades brasileiras no Leiden Ranking 2016, volume de artigos, citações e colaboração, e respectiva classificação global

\begin{tabular}{|c|c|c|c|c|c|c|c|c|c|c|}
\hline Univ & $\begin{array}{c}\mathrm{N} . \\
\text { artigos }\end{array}$ & Clas. & $\begin{array}{c}\text { PP(top } \\
10 \%) \\
\end{array}$ & Clas. & $\begin{array}{c}\text { PP(top } \\
50 \%) \\
\end{array}$ & Clas. & $\begin{array}{c}\mathbf{P P} \\
\text { (colab) }\end{array}$ & Clas. & $\begin{array}{l}\text { PP(int } \\
\text { colab) }\end{array}$ & Clas. \\
\hline USP & 26936 & 11 & $7,1 \%$ & 725 & $44,1 \%$ & 722 & $76,2 \%$ & 403 & $38,5 \%$ & 445 \\
\hline UNESP & 9396 & 192 & $5,6 \%$ & 818 & $40,7 \%$ & 806 & $77,5 \%$ & 334 & $32,2 \%$ & 558 \\
\hline UNICAMP & 9377 & 193 & $7,4 \%$ & 704 & $44,8 \%$ & 700 & $75,4 \%$ & 447 & $30,3 \%$ & 598 \\
\hline UFRJ & 8758 & 213 & $7,7 \%$ & 675 & $46,0 \%$ & 666 & $81,0 \%$ & 150 & $40,8 \%$ & 399 \\
\hline UFRGS & 7646 & 255 & $6,6 \%$ & 766 & $44,4 \%$ & 710 & $72,5 \%$ & 587 & $31,8 \%$ & 569 \\
\hline UFMG & 6322 & 316 & $6,6 \%$ & 764 & $44,9 \%$ & 696 & $79,2 \%$ & 245 & $35,9 \%$ & 490 \\
\hline UNIFESP & 5562 & 368 & $6,4 \%$ & 780 & $41,6 \%$ & 782 & $77,9 \%$ & 306 & $29,3 \%$ & 610 \\
\hline UFSC & 3623 & 531 & $7,2 \%$ & 713 & $46,3 \%$ & 653 & $76,6 \%$ & 382 & $34,2 \%$ & 515 \\
\hline UFPR & 3316 & 578 & $6,3 \%$ & 783 & $41,4 \%$ & 791 & $78,8 \%$ & 258 & $32,9 \%$ & 540 \\
\hline UFPE & 3163 & 598 & $5,6 \%$ & 822 & $40,5 \%$ & 811 & $78,4 \%$ & 270 & $31,9 \%$ & 563 \\
\hline UFSCAR & 2929 & 636 & $6,6 \%$ & 762 & $43,9 \%$ & 729 & $81,9 \%$ & 111 & $30,2 \%$ & 599 \\
\hline UNB & 2813 & 656 & $7,4 \%$ & 706 & $42,4 \%$ & 765 & $81,7 \%$ & 125 & $38,5 \%$ & 446 \\
\hline UFF & 2671 & 678 & $6,3 \%$ & 781 & $42,2 \%$ & 771 & $83,9 \%$ & 68 & $33,8 \%$ & 520 \\
\hline UFCE & 2620 & 687 & $7,4 \%$ & 703 & $46,5 \%$ & 645 & $84,0 \%$ & 64 & $34,9 \%$ & 502 \\
\hline UFV & 2191 & 764 & $5,1 \%$ & 833 & $38,9 \%$ & 826 & $75,4 \%$ & 448 & $27,7 \%$ & 654 \\
\hline UFSM & 2147 & 774 & $4,9 \%$ & 836 & $41,5 \%$ & 786 & $74,6 \%$ & 491 & $23,8 \%$ & 719 \\
\hline $\begin{array}{c}\text { Valores } \\
\text { mínimos } \\
\text { obtidos }\end{array}$ & 1349 & -- & $4,3 \%$ & -- & $35,9 \%$ & -- & $32,1 \%$ & -- & $10,7 \%$ & -- \\
\hline $\begin{array}{c}\text { Valores } \\
\text { máximos } \\
\text { obtidos } \\
\end{array}$ & 63936 & -- & $27,9 \%$ & -- & $72,0 \%$ & -- & $93,9 \%$ & -- & $89,3 \%$ & -- \\
\hline
\end{tabular}

Notas: Clas. : posição classificatória das universidades. Varia de 1 a 842.

PP Top 10\%: percentual das publicações entre as 10\% mais citadas na WoS

PP Top 50\%: percentual das publicações entre as 50\% mais citadas na WoS

PP Colab: percentual de publicações em colaboração institucional

Fonte: Leiden Ranking 2016

Observa-se que as universidades brasileiras ocupam posições globais bastante diversificadas no Leiden Ranking 2016, com exceção dos indicadores de impacto PP(top10\%) e PP(top50\%) - onde se observa a ocupação das últimas posições do ranking. A 
USP mantém sua hegemonia no que diz respeito ao volume de publicações, provavelmente reflexo de seu tamanho institucional - é a universidade que reúne o maior volume de professores contratados (GEOCAPES, 2016). No entanto, o destaque não é observado em relação ao impacto das suas publicações. Nesse sentido, a UFCE destaca-se com 7,4\% e $46,5 \%$ de publicações em Top $10 \%$ e Top $50 \%$ respectivamente, um percentual alto quando se considera que esta é uma das universidades com menor produção científica dentre as incluídas pelo Leiden Ranking.

0 percentual de colaboração institucional mantém-se num nível parecido entre as 16 instituições, variando de 72,5\% (UFRGS) a 84\% (UFCE). Já a colaboração internacional apresenta discrepância maior, variando de 23,8\% (UFSM) a 40,8\% (UFRJ).

Para contextualizar os resultados das universidades brasileiras, a últimas linhas da Tabela 1 apresentam os scores mínimo e máximo do Leiden Ranking. A Rockefeller University é líder em impacto, com $27,9 \%$ de suas publicações entre as top $10 \%$ e $72,0 \%$ entre as top50\%. 0 percentual de colaboração institucional é bastante elevado (93,9\%) na universidade líder nesse indicador, a China Medical University (Taiwan). A colaboração internacional é acentuada em universidades situadas em países de menor prestígio científico. A King Abdulaziz University (Arábia Saudita) apresenta 89,3\% de suas publicações nessa modalidade, e é apontada como exemplo de instituição que tenta burlar os rankings, contratando um grande número de professores cuja função principal é incluir a universidade como uma filiação secundária, a fim de coletar uma parte do crédito para publicações e citações (BORNMANN; BAUER, 2015).

Importante destacar que o Leiden Ranking baseia-se nas core publications da Web of Science, ou seja, artigos e artigos de revisão escritos em língua inglesa, assinados por um ou mais autores e publicadas em core journals. Por sua vez, são considerados core journals aqueles que possuem escopo internacional - medido através dos países de origem dos autores que publicam e citam o periódico; e que possuem um grande número de referências para outros core journals. Dessa forma, cerca de um sexto de publicações indexadas pela WoS que não atendem a estes requisitos são excluídas dos cálculos Leiden Ranking (CENTRE..., 2016). Entre as 146 revistas brasileiras presentes na lista de periódicos do Leiden Ranking 2016, apenas 25 (17\%) fazem parte do core journals. Portanto, os artigos nacionais que foram publicados nestas revistas não foram contabilizados como produção científica e citação das universidades brasileiras.

O Ranking de Shanghai inclui seis universidades brasileiras na edição 2016: USP, UFMG, UFRJ, UNESP, UFRGS e UNICAMP. Observa-se que a USP foi classificada entre as 150 melhores universidades do mundo, enquanto as outras brasileiras foram classificadas nas categorias seguintes: UFMG, UFRJ e UNESP entre a 301 e 400 melhores do mundo, e UFRGS e UNICAMP entre a 401 e 500. Os indicadores Alumni e Award não pontuam para nenhuma universidade brasileira tendo em vista que nenhuma delas recebeu prêmios Nobel ou Medalha Fields até a edição 2016 (Tabela 2).

Tabela 2 - Desempenho das universidades brasileiras no Ranking de Shanghai 2016

\begin{tabular}{llllllll}
\hline Univ & Classificação & $\begin{array}{l}\text { Alumni } \\
(10 \%)\end{array}$ & $\begin{array}{l}\text { Award } \\
(20 \%)\end{array}$ & $\begin{array}{l}\text { HiCi } \\
(20 \%)\end{array}$ & $\begin{array}{l}\text { N\&S } \\
(20 \%)\end{array}$ & $\begin{array}{l}\text { PUB } \\
(20 \%)\end{array}$ & $\begin{array}{l}\text { PCP } \\
(10 \%)\end{array}$ \\
\hline USP & $101-150$ & 0.0 & 0.0 & 10.3 & 12.6 & 73.3 & 16.5 \\
UFMG & $301-400$ & 0.0 & 0.0 & 10.3 & 4.2 & 38.0 & 19.5 \\
UFRJ & $301-400$ & 0.0 & 0.0 & 0.0 & 8.2 & 40.7 & 20.5 \\
UNESP & $301-400$ & 0.0 & 0.0 & 0.0 & 7.0 & 43.9 & 21.9 \\
UFRGS & $401-500$ & 0.0 & 0.0 & 0.0 & 5.5 & 38.7 & 19.3 \\
UNICAMP & $401-500$ & 0.0 & 0.0 & 0.0 & 3.7 & 41.6 & 20.6 \\
\hline $\begin{array}{l}\text { Maior } \\
\text { pontuação } \\
\text { obtida }\end{array}$ & -- & & & & & & \\
\hline $\begin{array}{l}\text { Menor } \\
\text { pontuação } \\
\text { obtida }\end{array}$ & -- & 100.0 & 100.0 & 100.0 & 100.0 & 100.0 & 100.0 \\
\hline
\end{tabular}

Fonte: ACADEMIC RANKING OF WORLD UNIVERSITIES (2016) 
O volume de publicação científica da USP resultou em 73.3 pontos de um total de 100 obtidos por Harvard. Ao analisar a pontuação obtida pela USP em PUB e compará-la com universidades internacionais que obtiveram pontuação semelhante neste indicador, é possível observar que as universidades internacionais alcançam em torno de 30 a 40 pontos nos indicadores $\mathrm{N} \& \mathrm{~S}$ (artigos nas revistas Nature e Science) e $\mathrm{HiCi}$ (pesquisadores ranqueados pelo Higly Cited), enquanto a USP pontuou 12.6 e 10.3 respectivamente, o que resulta em uma classificação geral inferior no score global. A ampliação da pontuação nestes indicadores nos próximos anos pode ser um fator definitivo para o posicionamento da USP entre as 100 primeiras universidades mundiais.

A alta pontuação obtida pela USP em publicações (PUB) a diferencia das outras universidades brasileiras, levando a formação de dois grupos distintos, um formado unicamente pela USP e outro que engloba as demais universidades. Já a situação das universidades brasileiras quanto ao indicador N\&S (artigos nas revistas Nature e Science) é mais homogênea. Visualiza-se um grupo único, apesar da pontuação variar de 3.7 (UNICAMP) a 12.6 (USP). Este é o indicador que pode fazer a diferença na classificação geral no Ranking de Shanghai, visto que cada artigo extra poderá resultar em avanços perceptíveis em termos de pontuação.

Junto com N\&S, o desempenho no indicador HiCi é importante para o Ranking de Shanghai: um único ponto neste indicador gera uma grande diferença para as universidades (DOCAMPO; CRAM, 2015; PIRO; SIVERTSEN, 2016). O indicador HiCi foi pontuado por USP e UFMG com um único pesquisador presente na lista do Highly Cited Researchers 2015: Paulo Artaxo, vinculado a USP na área de Geociências; e Ado Jorio de Vasconcelos, vinculado a UFMG na área de Física.

Com o objetivo de estimar o desempenho das 16 universidades presentes no Leiden Ranking nos parâmetros do Ranking de Shanghai, foram plotados no gráfico 1 o volume de artigos publicados na Web of Science e o número de artigos nas revistas Nature e Science:

Gráfico 1 - Estimativa de desempenho das universidades brasileiras no Ranking de Shanghai

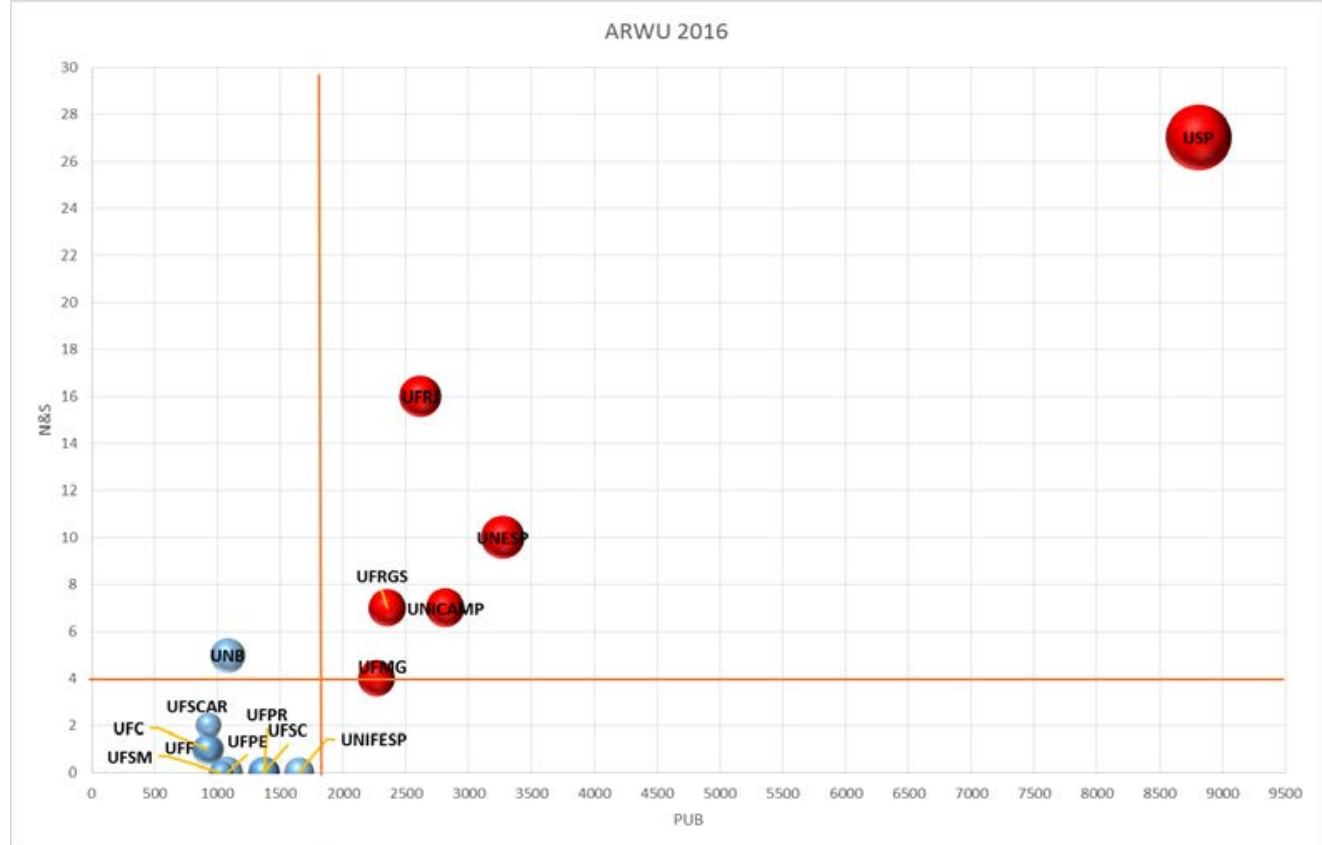

Fonte: Ranking de Shanghai e Web of Science

0 Gráfico 1 retrata o volume de artigos publicados na Web of Science (eixo x) e volume de artigos publicados nas revistas Nature e Science (eixo y). A contextualização dos pontos do Ranking de Shanghai pode apoiar algumas estratégias. A linha vertical do gráfico posiciona-se em torno de 1750 artigos na Web of Science enquanto a horizontal está posicionada em quatro artigos publicados na Nature e Science. A análise demonstra que, ao atender os dois requisitos, quais sejam, cerca de 1750 artigos publicados em revistas 
indexadas pela Web of Science e quatro artigos publicados nas revistas Nature e Science, a universidade atinge os requisitos para entrar no Ranking de Shanghai. Atender apenas um dos requisitos não dá acesso ao ranking. A UNB, por exemplo, apesar de possuir cinco artigos nas revistas Nature e Science, precisa ampliar o volume com cerca de 700 publicações na Web of Science. A UNIFESP também está próxima: o esforço para entrar equivale a cerca de 200 publicações na Web of Science e quatro artigos em Nature e Science.

O panorama no Ranking QS é mais abrangente, já que ele classificou 22 universidades brasileiras: USP, UNICAMP e UFRJ ocuparam, respectivamente, as posições 120, 191 e 321 na edição 2016 do ranking (Gráfico 2). As três universidades obtiveram alta pontuação nos indicadores Academic Reputation e Employer Reputation, responsáveis por $50 \%$ dos pontos totais do Ranking QS. Várias universidades brasileiras renomadas não pontuaram nestes indicadores devido aos métodos estabelecidos pelo Ranking, o que não significa que não tenham recebido nenhuma indicação de prestígio nas pesquisas de opinião com acadêmicos e empregadores.

Gráfico 2 - Pontuação obtida pelas universidades brasileiras no QS Ranking 2016

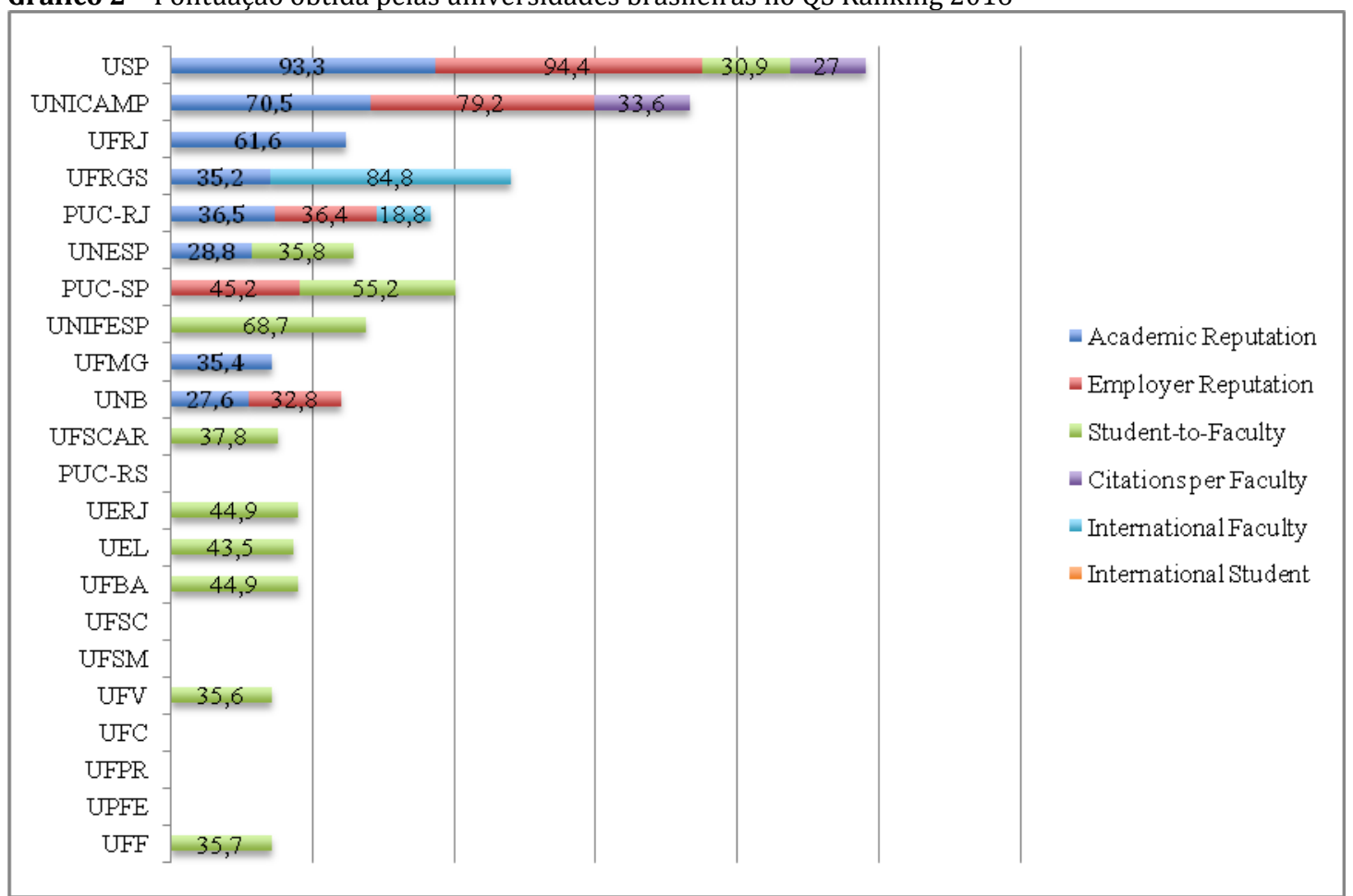

Nota: a pontuação varia de 1 a 100 para cada indicador.

Fonte: QS 2016

0 gráfico 2 auxilia a entender visualmente o desempenho das universidades brasileiras. Observa-se que USP e UNICAMP apresentam uma barra de pontuação mais alongada, demonstrando a obtenção de pontos em vários indicadores. Já a UFRJ apresenta pontuação somente no indicador Academic Reputation e, portanto, pode-se afirmar que seu posto como terceira melhor universidade brasileira no Ranking QS se deve unicamente a este indicador. 0 visibilidade da UFRJ pode estar atrelada ao fato de ser uma das universidades pioneiras, situada na primeira capital do Brasil e possivelmente, uma das cidades mais conhecidas internacionalmente e mais visitadas por estrangeiros. A PUC-RJ apresenta desempenho moderado, porém em três indicadores diferentes. As outras universidades apresentam pontuação em apenas dois ou um indicador, e algumas possuem pontuação zerada em todos os indicadores, indicando uma pontuação ainda muito baixa nestes indicadores.

0 indicador International Faculty não foi pontuado pelas universidades brasileiras, com exceção da UFRGS, que apresenta 84,8 pontos e da PUC-RJ, com 18,8 pontos. Mas sem dúvida o indicador International Student é o que mais deixa a desejar, tendo em vista que nenhuma universidade pontuou neste indicador. As causas podem ser muitas, começando 
pelo processo seletivo baseado em provas em língua portuguesa (vestibular ou ENEM), o que elimina ou minimamente dificulta o ingresso dos estrangeiros; até a dificuldade idiomática em aulas ministradas predominantemente em português. Na opinião de Lemos (2011), as universidades brasileiras estão isoladas do resto do mundo e estão, em termos de estudantes e professores, compostas inteiramente por brasileiros. Os baixos percentuais de internacionalização causam efeitos negativos nos rankings e também na capacidade para a inovação, já que a transferência de tecnologia não ocorre em universidades que têm como destinatária a população de seu entorno.

Chama atenção nos resultados a alta pontuação obtida no indicador Student-tofaculty, resultado do produto do número total de alunos dividido pelo número total de professores. Este resultado demonstra que grande parte das universidades brasileiras presentes no QS tem um número adequado de alunos por professor, o que é confirmado pelo Ranking THE apresentado a seguir. Outro resultado importante diz respeito ao indicador Citation per faculty, que só pontuou para USP e UNICAMP, reiterando os resultados do Leiden Ranking que demonstraram o baixo volume de citações recebidas pela produção científica das universidades brasileiras.

O Ranking THE é o mais inclusivo e classificou 27 universidades brasileiras em 2016 (Tabela 3):

3):

\begin{tabular}{|c|c|c|c|c|c|c|}
\hline \multirow[b]{2}{*}{ Univ } & \multirow[b]{2}{*}{ Classificação } & \multicolumn{5}{|c|}{ Indicadores Ranking THE e respectivo peso na pontuação total } \\
\hline & & $\begin{array}{l}\text { Citação } \\
(30 \%)\end{array}$ & $\begin{array}{l}\text { Indústria } \\
(2,5 \%)\end{array}$ & $\begin{array}{l}\text { Persp. } \\
\text { Intern. } \\
(7,5 \%)\end{array}$ & $\begin{array}{l}\text { Pesquisa } \\
(30 \%)\end{array}$ & $\begin{array}{l}\text { Ensino } \\
(30 \%)\end{array}$ \\
\hline USP & $251-300$ & 25.7 & 39.6 & 28.3 & 60.2 & 57.2 \\
\hline UNICAMP & $401-500$ & 28.0 & 46.5 & 24.1 & 39.6 & 44.9 \\
\hline UFABC & $601-800$ & 33.4 & 36.4 & 31.9 & 12.8 & 14.9 \\
\hline UFMG & 601-800 & 25.5 & 33.6 & 22.7 & 14.3 & 30.6 \\
\hline UFPR & $601-800$ & 8.0 & 32.1 & 17.2 & 23.9 & 24.3 \\
\hline UFRJ & $601-800$ & 20.1 & 41.7 & 27.9 & 15.7 & 31.1 \\
\hline UFRGS & $601-800$ & 23.9 & 32.1 & 21.5 & 10.8 & 31.0 \\
\hline UFSC & $601-800$ & 20.1 & 54.0 & 24.2 & 12.4 & 25.3 \\
\hline UNIFESP & $601-800$ & 26.8 & 32.1 & 22.3 & 19.1 & 37.5 \\
\hline PUC RJ & $601-800$ & 20.6 & 100.00 & 35.2 & 23.9 & 27.3 \\
\hline PUC RS & $601-800$ & 28.7 & 46.2 & 23.1 & 11.8 & 20.3 \\
\hline UNESP & $601-800$ & 9.2 & 34.5 & 18.8 & 18.8 & 29.5 \\
\hline UFBA & $801+$ & 14.4 & -- & 22.2 & 5.2 & 18.5 \\
\hline UFC & $801+$ & 16.6 & -- & 19.8 & 4.7 & 22.9 \\
\hline UFG & $801+$ & 14.0 & 32.1 & 18.9 & 7.1 & 17.4 \\
\hline UFOP & $801+$ & 13.2 & 33.9 & 17.2 & 4.6 & 14.5 \\
\hline UFPE & $801+$ & 11.5 & 32.1 & 18.5 & 5.0 & 23.5 \\
\hline UFRN & $801+$ & 10.6 & 44.0 & 18.5 & 8.2 & 20.9 \\
\hline UFSM & $801+$ & 7.2 & 32.8 & 14.5 & 5.0 & 20.3 \\
\hline UFSCAR & $801+$ & 12.9 & 32.1 & 21.4 & 6.8 & 23.0 \\
\hline UFLAVRAS & $801+$ & 5.1 & 43.7 & 14.9 & 14.2 & 31.9 \\
\hline UFV & $801+$ & 6.4 & 53.1 & 16.2 & 8.8 & 21.8 \\
\hline UFF & $801+$ & 8.5 & 32.3 & 22.7 & 7.9 & 18.4 \\
\hline UEL & $801+$ & 7.9 & 32.1 & 15.5 & 6.7 & 19.9 \\
\hline PUCPR & $801+$ & 24.6 & 33.9 & 19.3 & 8.0 & 14.4 \\
\hline UERJ & $801+$ & 17.8 & 49.2 & 17.8 & 10.8 & 20.9 \\
\hline UEM & $801+$ & 5.1 & 32.5 & 13.8 & 9.9 & 16.9 \\
\hline
\end{tabular}

Fonte: THE 2016 
USP e UNICAMP são as universidades com maior destaque no ranking, classificandose respectivamente entre as 251-300 e 401-500 melhores universidades, conforme a segunda coluna da Tabela 3. Os cinco indicadores THE estão apresentados nas colunas seguintes da Tabela 3, com respectivo peso atribuído. Para cada indicador, a pontuação varia de 1 ao máximo 100 obtida por Harvard (BOOKSTEIN et al., 2010). Os dados brutos não são disponibilizados pelo THE o que pode comprometer a possibilidade de benchmarking, tendo em vista que o entendimento das pontuações não é nada intuitivo (ELKEN; HOWDHAUGEN; STENSAKER, 2016).

A USP apresenta um desempenho destacado em Pesquisa e Ensino, indicador que alcança 60.2 e 57.2 pontos respectivamente, consequência do grande volume de produção científica e alta reputação da USP, a maior e possivelmente mais tradicional universidade brasileira. A reputação vincula-se ao tamanho e idade das instituições, tendo em vista que é construída ao longo dos anos de existência da instituição. 0 fato das universidades brasileiras serem novas pode explicar o baixo desempenho quando comparado a universidades pioneiras estabelecidas na Europa e nos Estados Unidos. Conforme apontado por Manganote, Schulz e Cruz (2016), o desempenho das universidades brasileiras no Ranking THE é influenciado positivamente pela produção e citação da Física de Altas Energias.

As universidades brasileiras em geral não apresentam boa performance no indicador Citação, resultado similar ao apresentado no Leiden Ranking e QS. Tendo em vista que este é um indicador que perfaz $30 \%$ do Ranking THE, o baixo desempenho das universidades brasileiras afeta bastante o posicionamento no ranking. Por outro lado, o indicador indústria revelou diversas universidades com desempenho superior a 50 pontos: PUC-RJ, UFSC e UFV.

\section{CONCLUSÕES}

Nas últimas décadas as universidades tornaram-se maiores, mais populares e mais globais, o que ajuda a explicar o interesse e influência dos rankings na atualidade. Porém, estabelecer comparações entre universidades não é um exercício simples, é preciso considerar variáveis como tamanho, história e contexto de criação, missão e objetivos. A avaliação das universidades através dos rankings é parcial tendo em vista que nenhum deles mensura todos os aspectos de uma universidade - os rankings utilizam indicadores diferentes, atribuindo pesos variados a cada um deles. Esta pesquisa oportunizou conhecer detalhes metodológicos sobre os principais rankings universitários globais e conhecer as críticas acerca de cada um deles, permitindo o entendimento aprofundado da informação contida em suas tabelas classificatórias.

Esta pesquisa buscou um retrato da situação das 29 universidades brasileiras classificadas nas edições 2016 dos rankings Leiden, Shanghai, QS e THE. Conclui-se que o desempenho das universidades brasileiras é muito bom, especialmente no que diz respeito ao alto volume da produção científica. Tal volume é responsável, junto com os artigos publicados nas revistas Nature e Science, pela presença de seis universidades brasileiras (USP, UNICAMP, UFMG, UFRJ, UFRGS e UNESP) no mais rigoroso deles, o Ranking de Shanghai. No Ranking Leiden estão presentes 16 universidades brasileiras. No QS, 22 universidades brasileiras. O THE, o mais abrangente deles, reúne 27 universidades brasileiras.

No entanto, o impacto, medido pelas citações recebidas, ainda constitui o principal desafio para os pesquisadores brasileiros. Algumas estratégias podem direcionar para resultados nesse sentido, como o incentivo à colaboração internacional, tendo em vista que este é um fator que amplia as citações. 0 apoio à publicação dos resultados de pesquisa em revistas de maior impacto também constitui uma estratégia fundamental, tendo em vista que estes veículos podem ser o caminho para maior visibilidade aos artigos.

As revistas de maior impacto são importantes também por compor o core journal do Leiden Ranking. A ampliação do volume de produção científica das universidades brasileiras em revistas que compõem o core journals é a estratégia básica para melhoria do posicionamento das mesmas neste ranking. 0 estímulo e o direcionamento de políticas 
editoriais para inclusão de revistas brasileiras em core journals também é uma medida que possibilitará ampliar a participação nacional no Leiden Ranking.

Muitas universidades brasileiras são contemporâneas e ainda estão em processo de construção de sua reputação e prestígio, o que poderá favorecer o resultado das futuras pesquisas de opinião feitas por rankings como THE e QS. Estratégias de apoio à colaboração científica internacional proporcionam o trânsito de pesquisadores e maior divulgação das universidades brasileiras no exterior. Ainda, o incentivo das atividades de convênio, mobilidades e recebimento de professores visitantes internacionais pode contribuir para que as universidades brasileiras se tornem reconhecidas internacionalmente, fazendo com que apareçam nas pesquisas de opinião que compõem os rankings THE e QS.

O financiamento das publicações na Nature e Science, tendo em vista que ambas operam com taxas de publicação (Article Processing Charge) pode ser usado como estratégia para incentivar o aumento do volume de artigos nestas revistas. 0 controle institucional das publicações na Nature e Science, especificamente no que diz respeito aos nomes de autores e filiação, também é uma estratégia fundamental, já que cada um dos artigos publicados nestas revistas desempenha um papel essencial e os problemas de padronização de nomes institucionais brasileiros são reconhecidos nas bases de dados Web of Science e SCOPUS. Conclui-se que a iniciativa mais simples e econômica deve partir das próprias universidades: dedicar-se a coletar e documentar as publicações da universidade, assegurando que metadados corretos e de qualidade sejam informados pelos pesquisadores nas publicações e consequentemente, estejam disponíveis aos rankings.

Financiamento: este projeto de pesquisa foi financiado pelo Conselho Nacional de Desenvolvimento Científico e Tecnológico (CNPq).

\section{REFERÊNCIAS}

ACADEMIC RANKING OF WORLD UNIVERSITIES. Disponível em: < http://www.shanghairanking.com>. Acesso em: 11 jul. 2016.

BOOKSTEIN, Fred L. et al. Too much noise in the Times Higher Education rankings. Scientometrics, Amsterdam, v. 85, p. 295-299, 2010.

BORNMANN, L.; MOYA-ANEGÓN, F. What proportion of excellent papers makes an institution one of the best worldwide? Specifying thresholds for the interpretation of the results of the SCImago Institutions rankings and the Leiden Ranking. Journal of the Association for Information Science and Technology, New York, v. 65, n. 4, p. 732-736, 2014.

BORNMANN, Lutz; BAUER, Johann. Which of the world's institutions employ the most highly cited researchers? An analysis of the data from highlycited.com. Journal of the Association for Information Science and Technology, New York, v. 66, n. 10, p. 2146-2148, 2015.

CASTRO, Cláudio Moura. Ciência e universidade. Rio de Janeiro: Jorge Zahar, 1986.

CENTRE FOR SCIENCE AND TECHNOLOGY STUDIES. Leiden Ranking. Disponível em: <www.leidenranking.com>. Acesso em: 01 jun. 2016.

DE FILIPPO, Daniela et al. Visibility in international rankings. Strategies for enhancing the competitiveness of Spanish universities. Scientometrics, Amsterdam, v. 93, p 949-966, 2012.

DOCAMPO, Domingo; CRAM, Lawrence. On the effects of institutional size in university classifications: the case of the Shanghai ranking. Scientometrics, Amsterdam, v. 102, p. 1325-1346, 2015.

ENSERINK, Martin. Who Ranks the University Rankers? Science, v. 317, n. 5841, 24 Ago. 2007, p. 1026-1028. [doi: 10.1126/science.317.5841.1026]

ELKEN, Mari; HOVDHAUGEN, Elisabeth; STENSAKER, Bjorn. Global rankings in the Nordic region: challenging the identity of research-intensive universities? Higher Education, Washington, Jan. 2016. Disponível em: < http://link.springer.com/article/10.1007/s10734-015-9975-6>. Acesso em: 02 jan. 2017.

FUNDAÇÃO DE AMPARO À PESQUISA DO ESTADO DE SÃO PAULO. Indicadores de ciência e tecnologia e inovação em São Paulo 2010. São Paulo: FAPESP, 2011.

FRANÇA, Carlos Marshal. Rankings universitários promovidos por jornais no espaço iberoamericano: El Mundo (Espanha), El Mercurio (Chile) e Folha de São Paulo (Brasil). 2015. 225 p. 
Dissertação (Mestrado em Educação). Pontifícia Universidade Católica de Campinas, Programa de PósGraduação Stricto Sensu em Educação, Campinas, 2015.

HAZELKORN, Ellen. How Rankings are Reshaping Higher Education. In: CLIMENT, V.; MICHAVILA, F.; RIPOLLÉS , M. (Eds.). Los rankings universitarios: mitos y realidades. Madrid: Técnos, 2013.

HICKS, Diana et al. The Leiden Manifesto for research metrics. Nature, London, v. 520, p. 429-431.

LEMOS, Ronaldo. Universities in Brazil are too closed to the world, and that's bad for innovation. 2011. Disponível em: < https://freedom-to-tinker.com/2011/06/15/universities-brazil-are-tooclosed-world-and-thats-bad-innovation/ >. Acesso em: 27 jan. 2017.

LETA, J.; GLÄNZEL, W.; THIJS, B. Science in Brazil. Part 2: sectoral and institutional research profiles. Scientometrics, Amsterdam, v. 67, n.1, p. 87-105, 2006.

MANGANOTE, Edmilson J.T.; SCHULZ, Peter A.; CRUZ, Carlos Henrique de Brito. Effect of high energy physics large collaborations on higher education institutions citations and rankings. Scientometrics, Amsterdam, v. 109, p. 813-826, 2016.

PEDROSA, Renato Hyuda de Luna; CHAIMOVICH, Hernan. Brazil. In: Relatório de ciência da Unesco: rumo a 2030: visão geral e cenário brasileiro. Paris: Unesco Publishing, 2015, 58 p. Disponível em: <

http://unesdoc.unesco.org/images/0023/002354/235407por.pdf>. Acesso em: 17 set. 2017.

PIRO, Fredrik Niclas; SIVERTSEN, Gunnar. How can differences in international university rankings be explained? Scientometrics, Amsterdam, v. 109, p. 2263-2278, 2016.

QS WORLD UNIVERSITY RANKING. Ranking 2016. Disponível em: <

http://www.topuniversities.com/university-rankings/world-university-rankings/2016>. Acesso em: 03 nov. 2016.

QS WORLD UNIVERSITY RANKING. INTELIGENCE UNIT. Methodology. 2016. Disponivel em: <http://www.iu.qs.com/university-rankings/indicator-academic/\#toggle-id-3>. Acesso em: 08 nov. 2016.

RAUHVARGERS, Andrejs. Global university rankings and their impact. European University Association, 2011. 85 p.

SAFON, Vicente. What do global university rankings really measure? The search for the $\mathrm{X}$ factor and $\mathrm{X}$ entity. Scientometrics, Amsterdam, v. 97, p. 223-244, 2013.

SANTOS, S. M. O; NORONHA, D.P. O desempenho das universidades brasileiras em rankings internacionais. Em Questão, Porto Alegre, v. 22, n. 2, p. 186-219, 2016.

SANZ-CASADO, E. etal. Rankings nacionales elaborados a partir de múltiples indicadores frente a los de índices sintéticos. Revista Española de Documentación Científica, Madrid, v. 36, n. 3, 2013.

SANZ-CASADO, E. (coord.). Guía de buenas prácticas para la participación de las universidades españolas en los rankings internacionales. Madrid: Ministerio de Educación, Cultura y Deporte. 2015.101 p.

SCHWARTZMAN, Simon. Um Espaço para a Ciência: a formação da comunidade científica no Brasil. Campinas: UNICAMP, 2015.

THE TIMES HIGHER EDUCATION RANKING. World University Rankings 2016-2017 Methodology. 2016. Disponível em: <https://www.timeshighereducation.com/world-universityrankings/methodology-world-university-rankings-2016-2017>. Acesso em: 23 nov. 2016.

VAN RAAN, Anthony F. J. Fatal attraction: conceptual and methodological problems in the ranking of universities by bibliometric methods. Scientometrics, Amsterdam, v. 62, n. 1, p. 133-143, 2005.

VOGEL, Michely Jabala Mamede. Como ler um ranking: a proposta do Brazilian Research Ranking. In: ENCONTRO NACIONAL DE PESQUISA EM CIÊNCIA DA INFORMAÇÃO, 15, 2014, Belo Horizonte. Anais...., Belo Horizonte: ANCIB, 2014. Disponível em: < http://enancib2014.eci.ufmg.br/documentos/anais/anais-gt7 >. Acesso em: 25 jan. 2017.

Editores do artigo: Enrique Muriel-Torrado, Edgar Bisset Alvarez, Camila Barros. 